\title{
Kawasaki disease: a review with emphasis on cardiovascular complications
}

\author{
Ricardo Duarte • Silvia Cisneros • Gabriel Fernandez • \\ Daniel Castellon • Cesar Cattani • Cíntia A Melo • \\ Asier Apocada
}

Received: 19 April 2010 /Revised: 4 July 2010 /Accepted: 12 July 2010 / Published online: 30 July 2010

(C) European Society of Radiology 2010

\begin{abstract}
Kawasaki disease (KD) is an acute systemic vasculitis that is currently the leading cause of acquired heart disease in childhood in the United States. Cardiovascular complications are the major cause of morbidity, are responsible for virtually all deaths from KD and should be evaluated as soon as possible after the acute phase to establish the baseline status, in order to predict disease progression and determine adequate treatment. In selected patients, electrocardiography (ECG)-gated cardiac computed tomography (CT) and magnetic resonance (MR) imaging are valuable non-invasive techniques that can be used to help diagnose the cardiovascular complications associated with KD. In this article, we review the epidemiology, aetiology and pathogenesis, histopathology, clinical features, cardiovascular complications and imaging, focusing on the role of cardiac CT and MR on the initial assessment and follow-up of the cardiovascular complications of $\mathrm{KD}$.
\end{abstract}

\footnotetext{
R. Duarte $(\bowtie)$

Department of Radiology, Centro Hospitalar Gaia/Espinho, Porto, Portugal

e-mail: guerra.duarte@gmail.com

S. Cisneros

Department of Radiology, Hospital Basurto,

Basurto, Spain

e-mail: silviacisneros@gmail.com

G. Fernandez

Department of Radiology, Hospital POVISA,

Vigo, Spain

e-mail: gabriel.fdez.perez@gmail.com

D. Castellon

Department of Radiology, Hospital POVISA,

Vigo, Spain

e-mail: danielcastellon@gmail.com
}

Keywords Kawasaki disease $\cdot$ Cardiovascular complications · Acute vasculitis

\section{Introduction}

Kawasaki disease (KD) is a self-limiting acute vasculitis that was first described in 1967 by Dr Tomisaku Kawasaki, a Japanese paediatrician. Although more than 40 years have passed since the original description of the clinical signs of this disease, the diagnosis remains challenging and the aetiology unclear.

This illness predominantly affects infants and young children of all ethnic backgrounds and is more frequent during winter and spring [1-3]. Nevertheless, it is clearly more prevalent in the Asian population [4]. In Japan, an annual incidence of 112 up to 175 cases per 100,000 children $<5$ years old has been reported, whereas in the

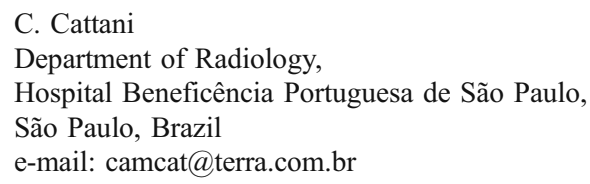

C. A Melo

Department of Radiology,

Hospital Beneficência Portuguesa de São Paulo,

São Paulo, Brazil

e-mail: camcat@terra.com.br
A. Apocada
Department of Pediatrics, Hospital Sant Joan de Déu, Barcelona, Spain 
United States an annual incidence of $20-25$ cases per 100,000 children $<5$ years old has been estimated [1-5]. There is a slightly increased prevalence in boys, with a male-to-female ratio of $1.3-1.7: 1$ and $76-80 \%$ of the children are $<5$ years old $[2,3]$.

A genetic predisposition has been suggested by higher rates of $\mathrm{KD}$ in the siblings as well as in the children of parents with a history of KD in childhood [1-5]. A recent study suggests that polymorphism in some alleles of B and $\mathrm{C}$ in HLA class I are associated with KD in Korean children [6].

Cardiovascular sequelae are the leading cause of morbidity and are responsible for virtually all deaths as a result of coronary vasculitis associated with a hypercoagulable state, leading to myocardial infarct and sudden death $[1-3,5]$. However, with proper treatment, the mortality rate is less than $1 \%$ in either Japan or the United States, with the peak mortality occurring between the 15 th and 45 th days $[2,4]$.

\section{Aetiology and pathogenesis}

The aetiology of KD remains unknown, although the clinical presentation-self-limiting illness manifested by an abrupt onset of fever, rash, exanthema, conjunctival injection and cervical adenopathy-and the epidemiological features - a seasonal peak in winter and spring, age distribution and a geographic wave-like spread of illness during epidemics-strongly suggest an infectious cause [1-4]. Nevertheless, after four decades of searching for a causal agent the researchers have failed to yield an infectious cause and have only reported a long list of pathogens that they have ruled out.

The hypothesis of a superantigen leading to upregulation of the immune response has been supported by some studies and refuted by others [1, 2, 4]. In a recent study, Leung et al. [7] failed to demonstrate a significant difference in the prevalence of toxin-producing strains between patients with KD and febrile controls.

An alternative hypothesis suggests the involvement of an oligoclonal antibody response for a conventional antigen, with the immunoglobulin A plasma cells playing a vital role [4].

Nevertheless, significant progress in finding the aetiology of KD is still expected in the near future.

\section{Histopathology}

$\mathrm{KD}$ is a systemic vasculitis that is more severe in small and medium arteries, and veins to a lesser extent, with inflammatory lesions in virtually all organs $[3,8]$. The earlier inflammatory changes are observed in the endothelium [1, 2, 4]. More advanced inflammatory lesions are consistent with oedema and inflammatory cell infiltrate in the subendothelial space, characterised by an influx of neutrophils in the first 10 days after onset with a rapid transition to mononuclear cells with lymphocytes (predominantly $\mathrm{CD} 8^{+} \mathrm{T}$ cells) $[1-3,5]$. In more severely affected vessels, the inflammatory lesions tend to progress leading to necrosis of smooth muscle cell media $[2,5]$. Destruction of internal and external elastic laminae may be seen in severely affected vessels. Loss of integrity of the wall vessel results from these changes, which may lead to the formation of aneurysms $[1,2,5]$.

Myocarditis, pericarditis and endocarditis with valvulitis may also be present in the acute phase [1, 3, 5].

After several weeks to months, the inflammatory changes of active vasculitis disappear and fibrous connective tissue, consisting of collagen and elastic fibres, begins to form within the vessel wall. The lesions in the coronary arteries continue to develop through an active process of remodelling characterised by myointimal proliferation and neoangiogenesis [1-5]. Over time the vessel may become stenotic or occluded, as a result of either progressive stenosis or superimposed thrombosis [5].

\section{Principal clinical features and diagnosis criteria}

The diagnosis of KD remains challenging, mainly because of the lack of a specific diagnostic test or clinical features. The diagnosis is based on the recognition of the classic clinical features of the illness, which include fever plus four of five other principal criteria (Table 1) $[1,2,8]$.

Fever is usually the first sign of KD and is classically high $\left(>39^{\circ} \mathrm{C}\right)$ and remittent [1-4]. With proper treatment, the fever usually resolves within 2-3 days, but without it may persist for several weeks, with an average of 11 days [1]. Typically, the other clinical signs appear over a period of several days ( $>5$ days).

Erythema and oedema of hands and feet confined to the palms and soles usually occurs in the acute phase, with the desquamation beginning in the periungual region within 10 20 days [1-3]. Deep transverse grooves across the nails (Beau's lines) may appear 1-2 months later [1,2].

The erythematous rash of KD may take various forms and is often non-specific [1-3]. Most common is a diffuse maculopappular erythematous rash (Fig. 1) [2]. The rash usually involves the trunk and extremities with predilection for the perineum $[1,2]$.

Bilateral conjunctival injection in $\mathrm{KD}$ is distinctive, usually involving the bulbar conjunctivae and is rarely exudative or painful [1-4]. 
Table 1 Diagnostic criteria for $\mathrm{KD}^{\mathrm{a}}$

\section{Criteria}

Fever persisting at least 5 days and the presence of at least four of the following five principal clinical features:

1. Changes in extremities:

Acute: erythema and oedema of hands and feet

Subacute: membranous desquamation of fingertips and periungual peeling

2. Polymorphous exanthema

3. Bilateral, painless bulbar conjunctival injection without exudate

4. Changes in lips and oral cavity: erythema and cracking of lips, strawberry tongue, diffuse injection of oral and pharyngeal mucosae

5. Cervical lymphadenopathy ( $>1.5 \mathrm{~cm}$ in diameter), usually unilateral

${ }^{\text {a }}$ Patients with fever at least 5 days and less than four principal symptoms can be diagnosed as having Kawasaki disease when coronary artery disease is detected. Other diseases with similar clinical features should be excluded

Changes in the lips and oral cavity include erythema, fissuring, peeling, cracking and bleeding of the lips, strawberry tongue and diffuse injection of oral and pharyngeal mucosae without associated mucosal ulcerations or pharyngeal exudates (Fig.1) [1-3].

Cervical lymphadenopathy is the least common of the main clinical features and is present in only $50-70 \%$ of the cases compared with other principal clinical features that are present in $90 \%$ of cases $[2,3]$. It is usually unilateral, nonsuppurative and limited to the anterior cervical triangle [2].

Recurrent episodes, defined as a new onset of illness after 3 months, can occur in $1 \%$ of the children [3].

Some patients with KD do not fulfil all the classic criteria for the clinical diagnosis. Children with fever and fewer than four of the other features of the illness present the so-called atypical or incomplete KD [9]. The atypical $\mathrm{KD}$ is also more common in young children and, as the recognition of this variant can be quite difficult, they are at greatest risk of developing coronary artery aneurysms (CAA) and fatal outcomes have occurred [2,9]. The diagnosis in these cases has usually been made through detection of CAA by echocardiography [2].

$\mathrm{KD}$ should be considered in the differential diagnosis of children with high fever of unexplained cause lasting more than 5 days with any of the principal clinical features of this disease, so that the proper treatment can be provided reducing the frequency and severity of cardiac complications. Nevertheless, as the diagnostic criteria are not specific to $\mathrm{KD}$, other diseases with similar clinical features should be excluded.
Fig. 1 A 3-year-old child with KD. Photographs show erythema, fissuring and bleeding of the lips (a) and diffuse maculopappular erythematous rash predominating the trunk and neck, with evident skin desquamation $(\mathbf{b}, \mathbf{c}, \mathbf{d})$

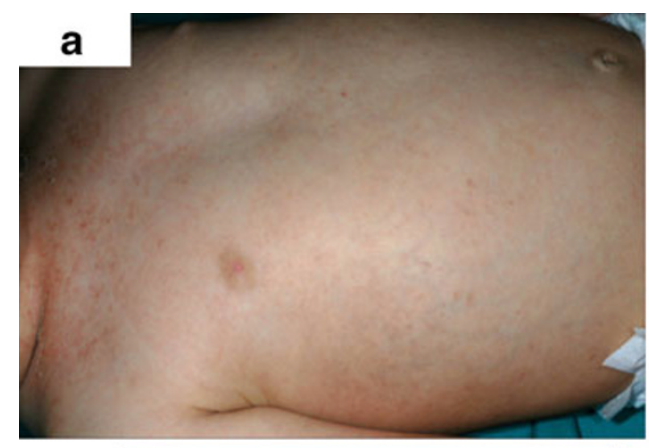

\section{b}

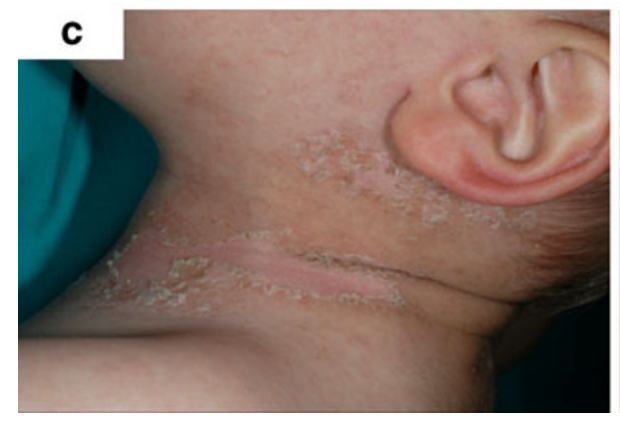

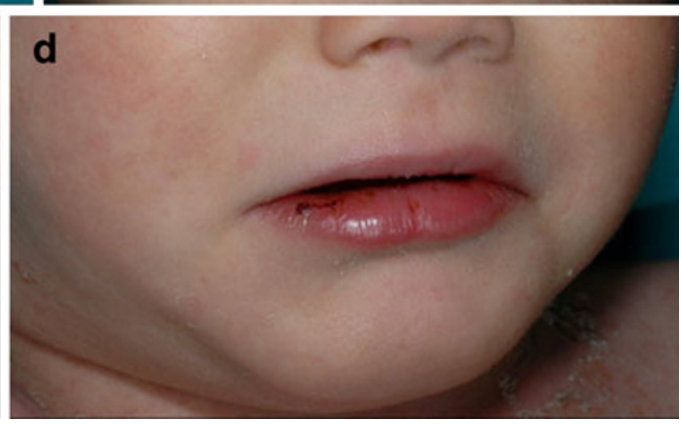


Table 2 Cardiovascular manifestations of KD

Manifestations (in order of frequency):

Coronary artery aneurysms

Myocarditis

Pericarditis with pericardial effusion

Systemic arterial aneurysms

Valvular disease

Mild aortic root dilatation

Myocardial infarct

\section{Associated features}

A series of other features are typical of $\mathrm{KD}$, although they are not included in the diagnostic criteria.

In the acute phase, the principal clinical features are typically accompanied by leukocytosis with neutrophil predominance and by elevated sedimentation rate and elevations in the levels of other acute-phase reactants [1-4]. Normocytic anaemia is also common in the acute phase [2].

A low platelet count at presentation has been associated with the development of severe coronary disease and myocardial infarction [10]. In later phases, usually after the second week, thrombocytosis is a characteristic finding $[2,3]$.

Mild to moderate elevations in serum transaminases and hypoalbuminaemia are also common findings [3].

Changes in chest radiographs are frequent. Umezawa et al. [11] found that $14.7 \%$ of children with $\mathrm{KD}$ had abnormal findings, including reticulogranular pattern in $89.5 \%$, peribronchial cuffing in $21.1 \%$, pleural effusion in $15.8 \%$, atelectasis in $10.5 \%$ and air trapping in $5.3 \%$.

At ultrasound examination, gallbladder hydrops (5\%), which is generally self-limiting, and enlarged kidneys with increased renal echoes and enhanced cortico-medullary differentiation may be seen [3].

\section{Cardiovascular complications and imaging}

$\mathrm{KD}$ is the leading cause of acquired heart disease in childhood in the United States. Cardiovascular complications are a major cause of morbidity (Table 2), the primary concern being CAA, which can develop in $15-25 \%$ of untreated children, usually in the subacute phase [1-5]. CAA are classified as small ( $<5 \mathrm{~mm}$ in internal diameter), medium $(5-8 \mathrm{~mm})$ or giant $(>8 \mathrm{~mm})$ [1]. CAA are classified as sacullar or fusiform (Fig. 2), are more frequently found in the proximal segments and at bifurcations and are often multiple (Fig. 3) [12]. The lesions in the coronary arteries seem to progress differently: the aneurysms in the right coronary artery (RCA) are more prone to massive thrombosis, whereas those in the left coronary artery (LCA) are more prone to progressive focal stenosis [13].

Angiographic spontaneous resolution may occur in about $50 \%$ of CAA, especially in the small coronary aneurysms, although the structural damage in the vessel wall persists $[3,14,15]$. The size of the aneurysm is a factor in prognosis, with giant aneurysms $(>8 \mathrm{~mm})$ being associated with a higher risk of rupture, thrombosis and stenosis, which can cause myocardial infarct and death [2, 13]. Giant aneurysms usually do not regress (Fig. 4) [16].

Several scoring systems have been developed in order to identify the risk factors for CAA, particularly giant CAA [17, 18]. Duration of fever has been consistently reported as a powerful risk factor [1, 17, 18]. Younger patient age, particularly less than 1 year of age, male sex and delayed diagnosis and treatment have also been associated with development of CAA [1, 17, 18]. Laboratory-detected conditions, including leukocytosis, thrombocytopaenia, lower haemoglobin or haematocrit and lower serum albumin, are also prominent risk factors $[7,17,18]$.

Treatment with a high dose of intravenous gamma globulin combined with aspirin has been proved to be

Fig. 2 A 5-year-old boy with $\mathrm{KD}$. Reconstructed volumerendered 3D CT coronary angiography (a, b) showing a fusiform aneurysm (arrow) involving the middle segment of the right coronary artery $(R C A)$
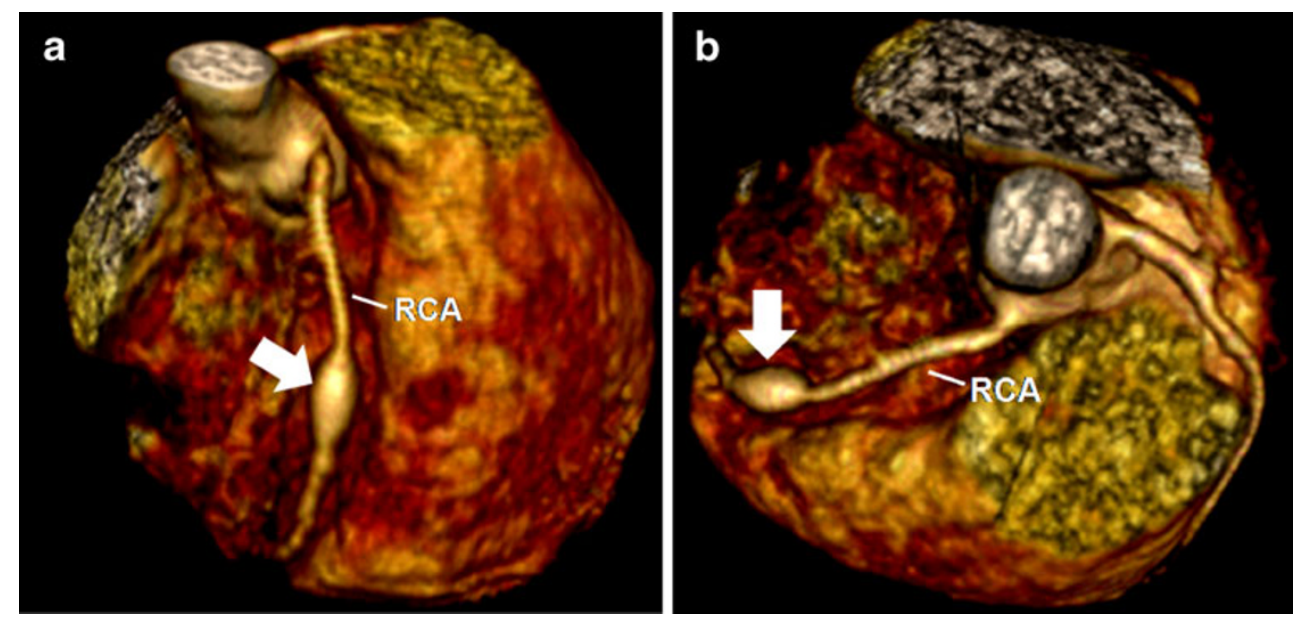

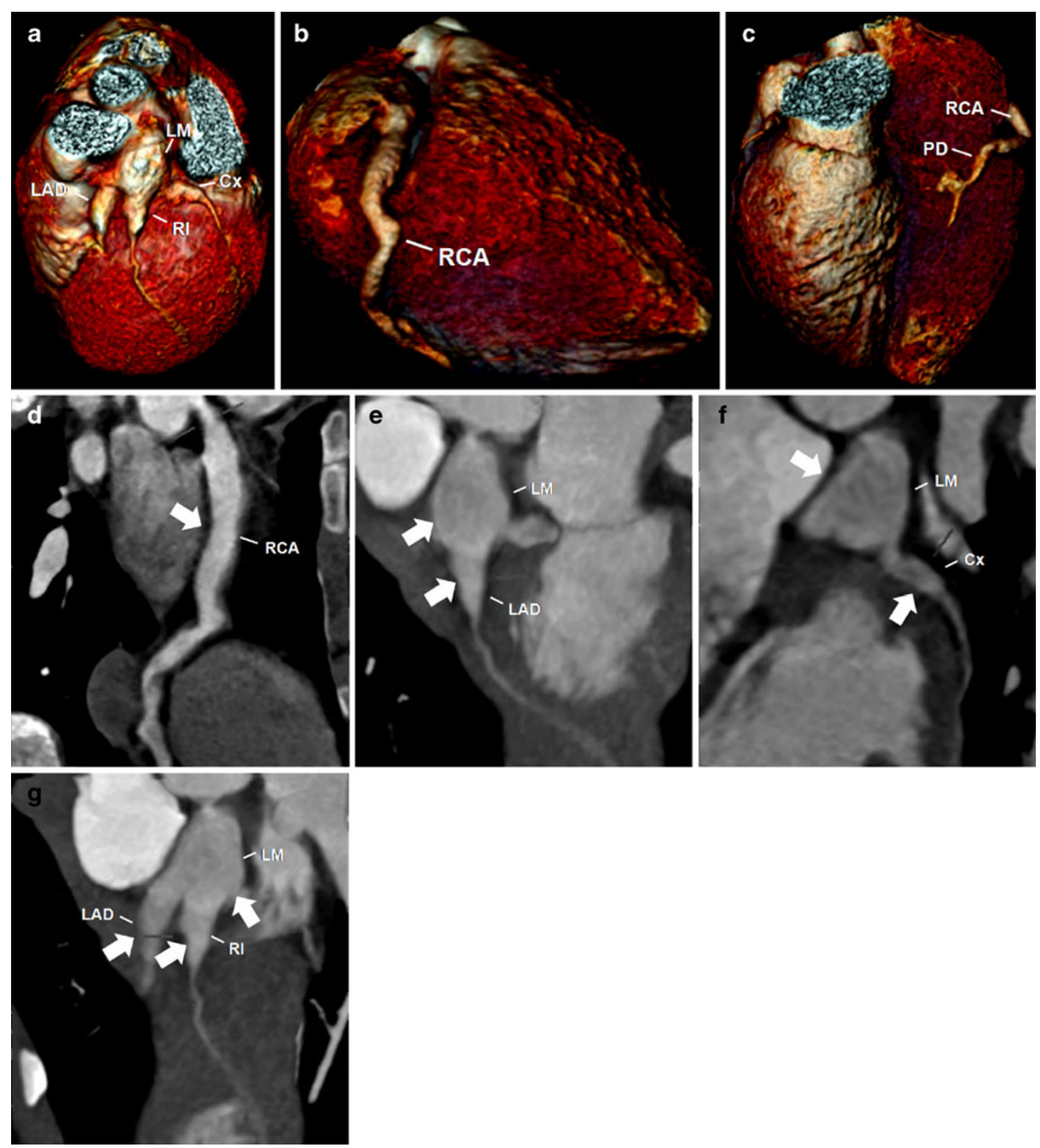

Fig. 3 A 5-year-old boy with KD. Reconstructed volume-rendered 3D CT coronary angiography $(\mathbf{a}, \mathbf{b}, \mathbf{c})$ revealing coronary arteries dilatations involving the right coronary artery $(R C A)$, including the posterior descending artery $(P D)$, the left main $(L M)$ and the proximal segments of the left anterior descending artery $(L A D)$ and circumflex artery $(C x)$ and the ramus intermedium $(R I)$. Maximumintensity projection $2 \mathrm{D}$ curved planar reformatting $\mathrm{CT}$ angiography

highly effective in reducing the clinical manifestations as well the prevalence of CAA to less than $5 \%$, although its mechanism of action remains unknown [19]. However, about $10 \%$ of patients do not respond favourably to this treatment, which represents an important clinical dilemma because of the increased risk of developing CAA [13].

The contribution of KD in childhood to the development of coronary artery disease in the long term is still

of the RCA (d) showing diffuse ectasia (arrow) affecting all segments, including the PD. Maximum-intensity projection 2D curved planar reformatting CT angiography of the left coronary artery (e, f, g) revealing a giant coronary without intraluminal thrombus of the LM and proximal segments of the LAD, Cx and RI (arrows)

controversial and remains a source of concern. Recent studies have shown that changes in lipid profile and endothelial dysfunction persist for a long time after the resolution of the illness [10, 20]. Noto et al. [21] recently demonstrated that patients with a history of KD have longterm structural and functional alterations that increase the propensity towards subclinical atherosclerosis with age. The current recommendation of the American Heart 
Fig. 4 A 40-year-old woman with a history of KD. Reconstructed volume-rendered 3D CT coronary angiography (a) demonstrating the left coronary artery with a giant fusiform aneurysm (arrow). Twodimensional curved planar reformatting CT angiography (b) showing a giant fusiform aneurysm (arrow) $9 \mathrm{~mm}$ in diameter in the left main $(L M)$ coronary artery and minimal mural calcifications (open arrows). MR coronary angiogram $(\mathbf{c}, \mathbf{d})$ and conventional coronary angiogram (e) showing the LM giant aneurysm (arrow). $L A D$ left anterior descending, $C x$ left circumflex, $R C A$ right coronary artery
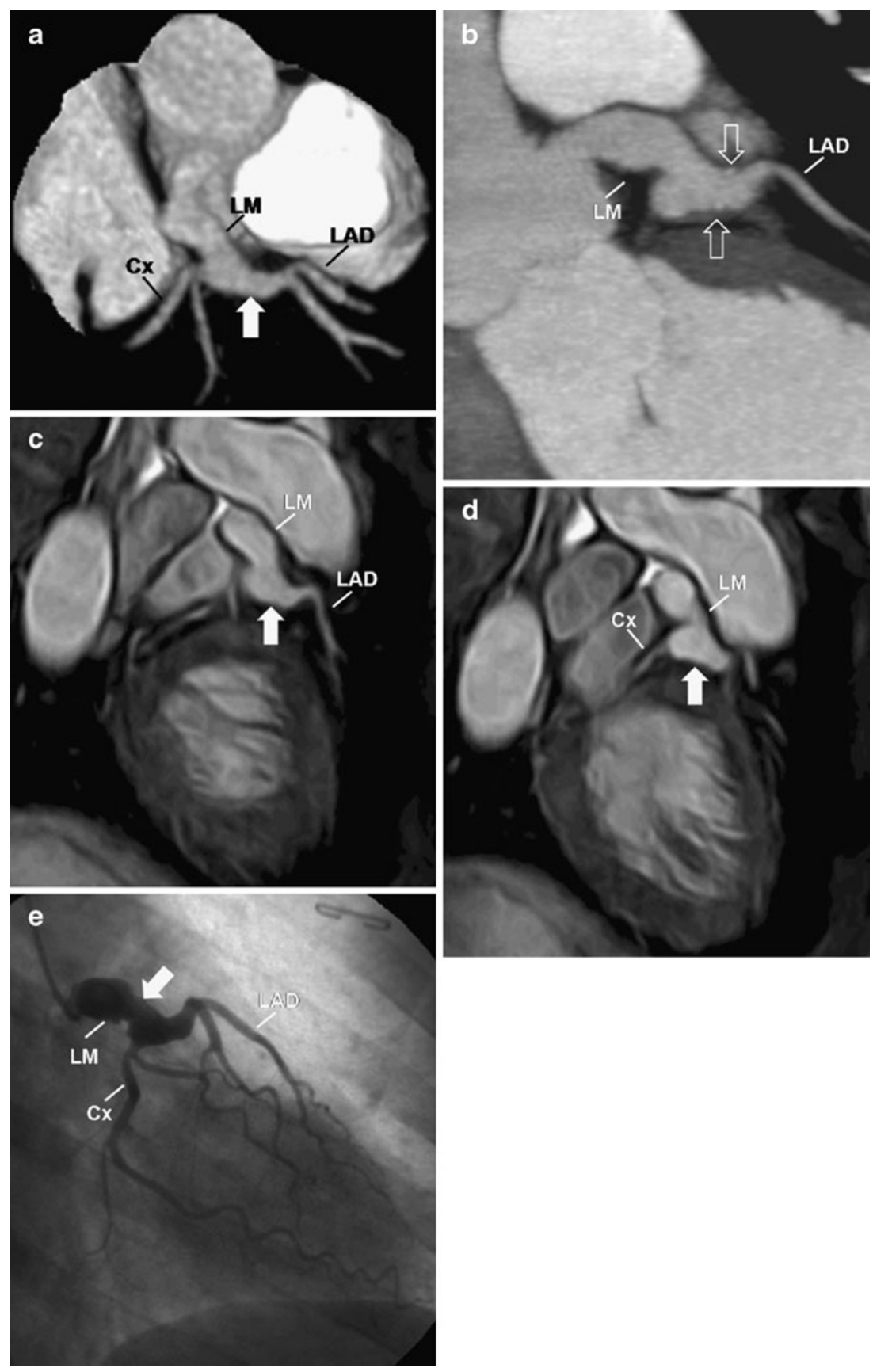

Association is to follow all patients with a history of $\mathrm{KD}$ [2].

Myocarditis is the most common non-coronary complication and is present in $50-70 \%$ of patients in the acute phase $[1,2]$. Pericarditis with pericardial effusion also frequently occurs in about $25 \%$ of patients in the acute phase [22]. Valvular disease, especially mitral regurgitation, may result from papillary muscle dysfunction or secondary to rupture of the chordae tendineae [3], myocardial infarct or valvulitis, and has been reported in about $1 \%$ of patients [22, 23]. Mild aortic root dilatation is also common in the acute phase and may be associated with aortic regurgitation [24].
The systemic arterial aneurysms occur in approximately $2 \%$ of patients with $\mathrm{KD}$, usually those who also have CAA [2]. The arteries most affected are the axillary, common iliac, internal iliac and subclavian (Fig. 5) [23]. The peripheral aneurysms also have a tendency to regress, similar to CAA. However, rarely serious complications may occur, such as severe peripheral ischaemia with resultant gangrene [2, 23].

Cardiac imaging is a critical part of the evaluation of all patients with KD disease as the major sequelae are related to CAA. The coronary anomalies should be evaluated as soon as possible after the acute phase to establish the initial 

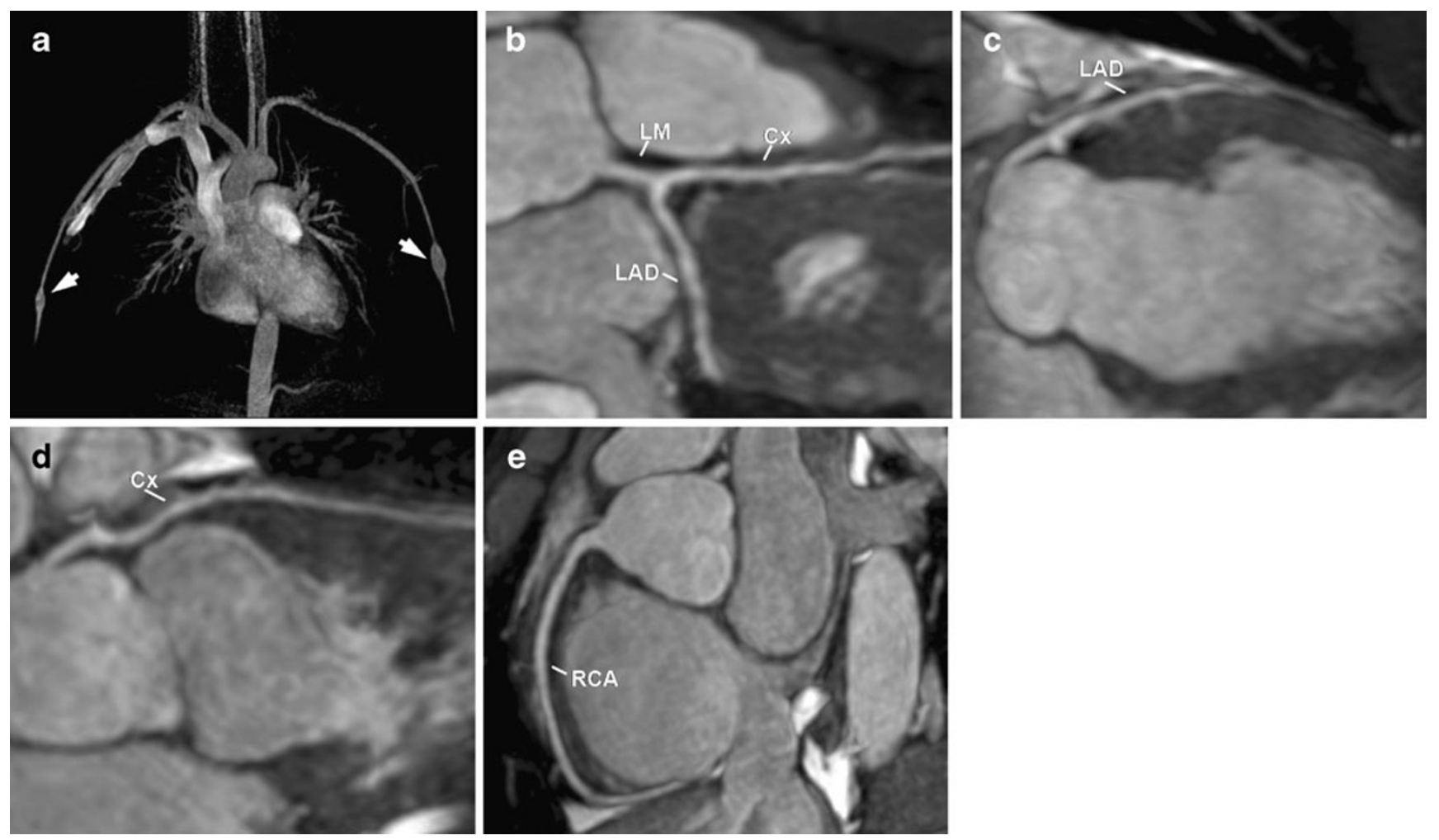

Fig. 5 A 16-year-old male patient with a history of KD. Reconstructed maximum-intensity-projection 3D MR angiography (a) demonstrating bilateral fusiform aneurysms in the brachial arteries

size and shape of the aneurysms in order to predict disease progression and determine adequate treatment. Evaluation of the coronary arteries should include quantitative assessment of the internal vessel diameters, as well as the number and location of the aneurysms, and absence or presence of intraluminal thrombus [1].

Echocardiography remains the technique of choice for the assessment of the coronary abnormalities because it is non-invasive, widely available and has high sensitivity and specificity for the detection of proximal abnormalities in the coronary tree $[1,2]$. However, the visualisation (arrows). MR coronary angiograms $(\mathbf{b}, \mathbf{c}, \mathbf{d}, \mathbf{e})$ with the maximumintensity-projection technique showing normal coronaries, without evidence of aneurysms

of coronary arteries cannot always be performed by echocardiography owing to technical limitations, such as limited field of vision or inadequate acoustic window, because as a child grows and the body size increases it becomes progressively more difficult or there are intrinsic limitations (inability to delineate the distal coronary segments or low sensitivity in demonstrating intraluminal thrombus). In these cases, other techniques-including angiography, computed tomography (CT) and magnetic resonance (MR) - may be of value for the assessment of coronary abnormalities.

Table 3 Comparison of coronary CT angiography and MR imaging in the diagnosis of KD

\begin{tabular}{llll}
\hline Technique & Advantages & Disadvantages & When to use \\
\hline $\begin{array}{l}\text { Coronary CT } \\
\text { angiography }\end{array}$ & $\begin{array}{l}\text { Fast } \\
\text { Wide availability } \\
\text { Excellent spatial resolution } \\
\text { High sensitivity and specificity for } \\
\text { detection of coronary abnormalities, } \\
\text { including distal segments }\end{array}$ & Uses ionising radiation & $\begin{array}{c}\text { Initial diagnostic work-up in patients } \\
\text { with inadequate acoustic window }\end{array}$ \\
& $\begin{array}{l}\text { No ionising radiation } \\
\text { MR imaging }\end{array}$ & $\begin{array}{c}\text { Relatively long examination } \\
\text { times }\end{array}$ & $\begin{array}{c}\text { Follow-up in patients with known CAA } \\
\text { and with inadequate acoustic window }\end{array}$ \\
& Excellent temporal resolution & Low spatial resolution & $\begin{array}{c}\text { Potential role as complementary examination } \\
\text { in the assessment of cardiovascular } \\
\text { non-coronary complications }\end{array}$ \\
\hline
\end{tabular}


Although selective coronary angiography remains the "gold standard" for the assessment of coronary abnormalities, its invasiveness, high cost, the need for hospitalisation and potential complications (haematomas, dissections, vascular rupture, arrhythmias and death) [25]—especially because most patients with $\mathrm{KD}$ are children-should be weighed against the expected benefits.

In selected patients, CT and MR are attractive noninvasive alternatives for the initial evaluation of coronary abnormalities, as well as for the surveillance of coronary abnormalities, as both are efficient at detecting CAA.

Several recent studies have demonstrated the feasibility of coronary CT angiography for the assessment of coronary artery disease with high diagnostic accuracy: high negative predictive values ranging from 95 to $100 \%$ [26-30]. Coronary CT angiography with ECG-gating is fast, widely available and offers excellent spatial resolution, allowing the assessment of virtually all coronary segments, including the distal segments, thus providing an accurate characterisation or a confident exclusion of CAA, as well as of the presence of an intraluminal thrombus (Fig. 4).

However, as this illness affects mainly children, the dose of radiation remains a major concern. Nevertheless, several strategies to reduce radiation exposure have been developed, including tube modulation, the use of lower tube voltages and of prospective electrocardiographic (ECG) triggering, allowing the reduction of effective radiation dose to the range of 1.3-4.2 mSv [31-34]. Recent studies have demonstrated that the use of prospective ECG-triggered high-pitch spiral acquisition using dual-source $\mathrm{CT}$ can reduce even more the effective radiation dose to less than $1 \mathrm{mSv}$ [35-37].

Magnetic coronary angiography is a desirable non-invasive technique not only because of the lack of ionising radiation but also because it allows the simultaneous assessment of coronary and non-coronary abnormalities. Several studies have reported a $100 \%$ agreement for the diagnosis of CAA between MR coronary angiography and selective coronary angiography in patients with $\mathrm{KD}$, with excellent agreement for diameter and length measurements [38-40]. Takemura et al. [41] demonstrated the utility and feasibility of coronary MR angiography for the evaluation of CAA, including in the demonstration of intraluminal thrombus, even in small children. MR is already accepted as the "gold standard" for the assessment of LV systolic function, which also allows the assessment of associated non-coronary cardiovascular complications, such as myocarditis or myocardial infarct. MR may play a potential role in the acute phase when myocarditis is suspected. MR imaging findings that are suggestive of myocarditis include abnormal signal intensity on STIR-weighted sequences in the affected regions, visible as an area of hyperintensity and regions of delayed contrast enhancement affecting the subepicardial myocardium in a patchy distribution, frequently sparing the subendocardium and without correlation with a vascular territory, allowing the differentiation from myocardial infarct [42, 43]. A similar pattern of enhancement may also be present in the pericardium when it is also involved in the inflammatory process [42]. Delayed gadolinium enhancement may be useful to help determine myocardial viability. MR may also be used to evaluate peripheral artery aneurysms. The advantages and disadvantages of coronary CT angiography and MR imaging are summarised in Table 3.

\section{Conclusion}

As cardiovascular sequelae are a major factor in morbidity and mortality cardiovascular imaging plays a vital role in the evaluation of all patients with KD. Although echocardiography remains the primary imaging technique in the evaluation of cardiovascular complications, especially in the acute phase, in selected patients cardiac CT and MR allow a non-invasive and reliable assessment of cardiovascular complications, as well as at follow-up, especially after childhood. Invasive coronary angiography should be reserved for patients with more complex coronary lesions or for therapeutic measures.

\section{References}

1. Newburger JW, Takahashi M, Gerber MA, Gewitz MH, Tani LY, Burns JC et al (2004) Diagnosis, treatment, and long-term management of Kawasaki disease: a statement for health professionals from the Committee on Rheumatic Fever, Endocarditis and Kawasaki Disease, Council on Cardiovascular Disease in the Young, American Heart Association. Circulation 110:27472771

2. Rowley AH, Shulman ST (1998) Kawasaki syndrome. Clin Microbiol Rev 11:405-414

3. Chung C, Stein L (1998) Kawasaki disease: a review. Radiology 208:25-33

4. Burns JC (2007) The riddle of Kawasaki disease. N Engl J Med 356:659-661

5. Burns JC, Kushner HI, Bastian JF, Shike H, Shimizu C, Matsubara T, Turner CL (2000) Kawasaki disease: a brief history. Pediatrics 106:E27

6. Oh JH, Han JW, Lee SJ, Lee KY, Suh BK, Koh DK et al (2008) Polymorphisms of human leukocyte antigen genes in Korean children with Kawasaki disease. Pediatr Cardiol 29:402-408

7. Leung DY, Meissner HC, Shulman ST, Mason WH, Gerber MA, Glode MP et al (2002) Prevalence of superantigen-secreting bacteria in patients with Kawasaki disease. J Pediatr 140:742-746

8. Ozen S, Ruperto N, Dillon MJ, Bagga A, Barron K, Davin JC et al (2006) EULAR/PReS endorsed consensus criteria for the classification of childhood vasculitides. Ann Rheum Dis 65:936-941

9. Rowley AH, Gonzalez-Crussi F, Gidding SS, Duffy CE, Shulman ST (1987) Incomplete Kawasaki disease with coronary artery involvement. J Pediatr 110:409-413

10. Newburger JW, Burns JC, Beiser AS, Loscalzo J (1991) Altered lipid profile after Kawasaki syndrome. Circulation 84:625-631 
11. Umezawa T, Saji T, Matsuo N, Odagiri K (1989) Chest x-ray findings in the acute phase of Kawasaki disease. Pediatr Radiol 20:48-51

12. Petrunić M, Drinković N, Stern-Padovan R, Mestrović T, Lovrić D (2009) Thoracoabdominal and coronary arterial aneurysms in a young man with a history of Kawasaki disease. J Vasc Surg 50:1173-1176

13. Suzuki A, Kamiya T, Yasuo O, Kuroe K (1991) Extended long term follow-up study of coronary arterial lesions in Kawasaki disease. J Am Coll Cardiol 17:33A

14. Fukushige J, Takahashi N, Ueda K, Hijii T, Igarashi H, Ohshima A (1996) Long-term outcome of coronary abnormalities in patients after Kawasaki disease. Pediatr Cardiol 17:71-76

15. Khositseth A, Siripornpitak S, Pornkulr R, Wanitkun S (2008) Giant coronary aneurysm caused by Kawasaki disease: follow-up with echocardiography and multidetector $\mathrm{CT}$ angiography. Br J Radiol 81:106-109

16. Inoue $\mathrm{O}$, Akagi $\mathrm{T}$, Kato $\mathrm{H}$ (1980) Fate of giant coronary artery aneurysms in Kawasaki disease: long-term follow-up study. Circulation 80:262

17. Sabharwal T, Manlhiot C, Benseler SM, Tyrrell PN, Chahal N, Yeung RS, McCrindle BW (2009) Comparison of factors associated with coronary artery dilation only versus coronary artery aneurysms in patients with Kawasaki disease. Am J Cardiol 104:1743-1747

18. McCrindle BW, Li JS, Minich LL, Colan SD, Atz AM, Takahashi $M$, Vetter VL et al (2007) Coronary artery involvement in children with Kawasaki disease: risk factors from analysis of serial normalized measurements. Circulation 116:174-179

19. Durongpistkul D, Gururaj V, Park J, Martin CF (1995) The prevention of coronary artery aneurysm in Kawasaki disease: a meta-analysis on the efficacy of aspirin and immunoglobulin treatment. Pediatrics 96:1057-1061

20. Cabana VG, Gidding SS, Getz GS, Chapman J, Shulman ST (1997) Serum amyloid A and high density lipoprotein participate in the acute phase response of Kawasaki disease. Pediatr Res 42:651-655

21. Noto N, Okada T, Karasawa K, Ayusawa M, Sumitomo N, Harada K, Mugishima H (2009) Age-related acceleration of endothelial dysfunction and subclinical atherosclerosis in subjects with coronary artery lesions after Kawasaki disease. Pediatr Cardiol 30:262-268

22. Gidding SS (1987) Late onset valvular dysfunction in Kawasaki disease. Prog Clin Biol Res 250:305-309

23. Kato H, Sugimura T, Akagi T, Sato N, Hashino K, Maeno Y et al (1996) Long-term consequences of Kawasaki disease. A 10- to 21year follow up study of 594 patients. Circulation 94:1379-1385

24. Ravekes WJ, Colan SD, Gauvreau K, Baker AL, Sundel RP, van der Velde ME et al (2001) Aortic root dilation in Kawasaki disease. Am J Cardiol 87:919-922

25. Kennedy JW, Baxley WA, Bunnel IL, Gensini GG, Messer JV, Mudd JG et al (1982) Mortality related to cardiac catheterization and angiography. Catheter Cardiovasc Diagn 8:323-340

26. Abdulla J, Abildstrom SZ, Gotzsche O, Christensen E, Kober L, Torp-Pedersen C (2007) 64-multislice detector computed tomography coronary angiography as potential alternative to conventional coronary angiography: a systematic review and metaanalysis. Eur Heart J 28:3042-3050

27. Vanhoenacker PK, Heijenbrok-Kal MH, Van Heste R, Decramer I, Van Hoe LR, Wijns W, Hunink MG (2007) Diagnostic performance of multidetector CT angiography for assessment of coronary artery disease: meta-analysis. Radiology 244:419-428

28. Hamon M, Morello R, Riddell J, Hamon M (2007) Coronary arteries: diagnostic performance of 16- versus 64-section spiral CT compared with invasive coronary angiography: meta-analysis. Radiology 245:720-731
29. Pugliese F, Mollet NR, Hunink MG, Cademartiri F, Nieman K, van Domburg RT et al (2008) Diagnostic performance of coronary CT angiography by using different generations of multisection scanners: single-center experience. Radiology 246:384-393

30. Pugliese F, Mollet NR, Runza G, van Mieghem C, Meijboom WB, Malagutti P et al (2005) Diagnostic accuracy of non-invasive 64-slice CT coronary angiography in patients with stable angina pectoris. Eur Radiol 16:575-582

31. Hirai N, Horiguchi J, Fujioka C, Kiguchi M, Yamamoto H, Matsuura N et al (2008) Prospective versus retrospective ECGgated 64-detector coronary CT angiography: assessment of image quality, stenosis, and radiation dose. Radiology 248:424-433

32. Shuman WP, Branch KR, May JM, Mitsumori LM, Lockhart DW, Dubinsky TJ et al (2008) Prospective versus retrospective ECG gating for 64-detector CT of the coronary arteries: comparison of image quality and patient radiation dose. Radiology 248:431-440

33. Earls JP, Berman EL, Urban BA, Curry CA, Lane JL, Jennings RS et al (2008) Prospectively gated transverse coronary CT angiography versus retrospectively gated helical technique: improved image quality and reduced radiation dose. Radiology 246:742-753

34. Herzog BA, Husmann L, Burkhard N, Gaemperli O, Valenta I, Tatsugami F et al (2008) Accuracy of low-dose computed tomography coronary angiography using prospective electrocardiogram-triggering: first clinical experience. Eur Heart J 29:3037-3042

35. Achenbach S, Marwan M, Ropers D, Schepis T, Pflederer T, Anders $\mathrm{K}$ et al (2010) Coronary computed tomography angiography with a consistent dose below $1 \mathrm{mSv}$ using prospectively electrocardiogram-triggered high-pitch spiral acquisition. Eur Heart J 31:340-346

36. Leschka S, Stolzmann P, Desbiolles L, Baumueller S, Goetti R, Schertler T et al (2009) Diagnostic accuracy of high-pitch dualsource CT for the assessment of coronary stenoses: first experience. Eur Radiol 19:2896-2903

37. Lell M, Marwan M, Schepis T, Pflederer T, Anders K, Flohr T et al (2009) Prospectively ECG-triggered high-pitch spiral acquisition for coronary $\mathrm{CT}$ angiography using dual source $\mathrm{CT}$ : technique and initial experience. Eur Radiol 19:2576-2583

38. Suzuki A, Takemura A, Inaba R, Sonobe T, Tsuchiya K, Korenaga $\mathrm{T}$ (2006) Magnetic resonance coronary angiography to evaluate coronary arterial lesions in patients with Kawasaki disease. Cardiol Young 16:563-571

39. Mavrogeni S, Papadopoulos G, Douskou M, Kaklis S, Seimenis I, Baras P et al (2004) Magnetic resonance angiography is equivalent to X-ray coronary angiography for the evaluation of coronary arteries in Kawasaki disease. J Am Coll Cardiol 43:649652

40. Arnold R, Ley S, Ley-Zaporozhan J, Eichhorn J, Schenk JP, Ulmer H, Kauczor HU (2007) Visualization of coronary arteries in patients after childhood Kawasaki syndrome: value of multidetector $\mathrm{CT}$ and MR imaging in comparison to conventional coronary catheterization. Pediatr Radiol 37:998-1006

41. Takemura A, Suzuki A, Inaba R, Sonobe T, Tsuchiya K, Omuro M, Korenaga T (2007) Utility of coronary MR angiography in children with Kawasaki disease. AJR Am J Roentgenol 188:534539

42. Friedrich MG, Sechtem U, Schulz-Menger J, Holmvang G, Alakija P, Cooper LT et al (2009) Cardiovascular magnetic resonance in myocarditis: A JACC White Paper. J Am Coll Cardiol 53:1475-1487

43. Mahrholdt H, Goedecke C, Wagner A, Meinhardt G, Athanasiadis A, Vogelsberg H et al (2004) Cardiovascular magnetic resonance assessment of human myocarditis: a comparison to histology and molecular pathology. Circulation 109:1250-1258 